\title{
Aldo Bressan \\ SOME ANSWERS TO MAX URCHS' COMMENTS ON A PAPER BY A. BRESSAN
}

1. Introduction. ${ }^{*}$ In [13] M. Urchs comments on my paper [5]. I think these comments are very useful, especially because (on p. 38) the author decides to "proceed with some methodological remarks concerning Bressan's account and his underlying attitude towards logical formalization in general", and thus he refers not only to [5].

In sect. 2 I mainly discuss a critical remark to [5], which in my opinion is due (perhaps completely) to a misunderstanding concerning the interpretation of $\diamond$; and in this connection I explain my disagreement with the use of "formal counterparts for" in Urchs assertion in [13, p 38]

(A) [A. Bressan] seeks to construct a logical calculus which allows the definition of formal counterparts for basic notions of the language of contemporary physical theory.

In sect. $2 \diamond$ 's intended interpretation is briefly hinted at; but in sect. 3 some considerations, in part new, are added, mainly because $\diamond$ is used in [5] within theories of general relativity.

In $[13$, pp. 38,40$]$ Urchs says that

(B) [the question] whether Bressan has succeeded in his effort or not

$[\ldots]$ shall be answered by the physicists.

and that

$\left(B^{\prime}\right)$ [this] can be authoritatively evaluated only by physicists (including, of course, Bressan himself), making use (or not) of the accom-

* The present work has been performed in the activity sphere of the Consiglio Nazionale della Ricerche. 
plished definitions. We thus arrive at the classical situation when logical formalization [...] becomes ontologically creative.

By the last part of $\left(B^{\prime}\right)$ I incidentally note that some recent works constitute an example showing that, independently of my above disagreement, the definitions referred to in $(A)$ - or in other words the foundations of the Mach-Painlevé type (for physical theories) — can be useful for scientific purposes that are unrelated to foundations and can interest experimenters. ${ }^{1}$

In [13, pp. 40-41] Urchs in effect notes that:

$\left(B^{\prime \prime}\right)$ the "proof of reality" of Bressan's construction is missing, i.e. one does not know whether this construction is accepted by scientists working in fields where it is supposed to function.

In sect. 4 a possible answer, in my opinion, is given to the question mentioned in $(B)$, also referring to

(i) the "proof of reality", even if "[today Urchs is not sure whether, e.g. his objection $\left(B^{\prime \prime}\right)$ was] to the point" - see [13, p. 41] — and to

(ii) an example in biology of the acceptance of Bressan's formal system presented in [2].

2. For the afore-mentioned discussion related to $\diamond$ 's interpretation, and for some brief remarks, I quote assertions $(C)$ to $(F)$ below from [13, p. 38]:

$(C)$ Another important problem is the implementation of an appropriate concept of causality, i.e. a formal explication of causal nexus [...]

(D) [Bressan's] formal framework contains no explicit definition of causality.

${ }^{1}$ E.g. the papers (labeled) [38], [41] and [192] to [193] in (the references of paper) [4] were mainly written as contributions to the foundations of the classical theory of continuous media; and the foundations referred to in the above papers are of the Mach-Painlevé type,in the rigorous setting worked out in [1] using modal notions and, e.g., possibilily axioms - see, e.g., [4, Ftn. 1].

In the works [9] (a)-(b) and [6], not yet appeared, it is emphasized that (briefly):

(i) e.g., several of the above contributions show that (a) for instance two conceivable thermo-elastic bodies $B$ and $B^{\prime}$ are globally equivalent i.e. (roughly speaking) they behave in the same way, with respect to some one-to-one correspondence $K$ between their material points, along processes not involving cuts, while (b) some $K$-corresponding subbodies of them fail to be globally equivalent w.r.t. $K$; and that

(ii) some among the same contributions treat in effect some natural problems related to couples of globally equivalent bodies.

Furthermore, [6] aims at rendering the physical implementation of such couples of bodies easier. 
(E) Bressan decided to choose the natural (modally) absolute notions of mass point, matter portion, and event points as primary. ${ }^{2}$

$(F)$ But anyway, one can hardly do without any formal counterpart of causal nexus.

The subsequent aforementioned criticism in [13] seems to be essentially based on $(D)$ to $(F)$. In fact

$\alpha)$ I strongly agree with $(F)$. However,

$\beta$ ) In [5, Ftn. 4, p. 25] it is asserted that the possibility notion used in [5] is in effect the one of physical possibility ${ }_{1}$ explained in [3] mainly within $[. .$.$] classical space-time (the above index "1" to be read as "in the first$ sense" is lacking in [5], Ftn. 4).

Furthermore the Ftns. 1 to 4 (and 6) in paper [4], which together with [5] constitutes one work, are devoted to physical possibility; in particular [4, Ftn. 4] aims at giving a brief explanation of this notion in the space-time of general relativity.

As will be explained in more detail in sect. 3,

$\gamma)$ in [3] and [4, Ftn. 1] causal possibility ${ }_{r}$ is, in effect, intuitively used in various senses distinguished by $r$ 's values; and physical possibility $r_{r}$ is its restriction to physical propositions. Thus

$\delta)$ paper [3] affords a formal counterpart of causal nexus for my axiomatic systems, such as the one mentioned in $(F)$; this is in effect " $\diamond$ " meant as "it is physically [or causally] possible,".

In connection with $(A)$ I note that the basic notions that Bressan (or Mach and Painlevé) decided to define are, for instance, mass and force in classical physics, and admissible space-time frame as well as space-time metric in theories of general relativity. I regard these definienda to be notions (and not formal counterparts for notions), whether or not they are used as primitives, as well as ordinary defined notions; the more so as

$\epsilon)$ an axiom of physical (hence causal) possibility is essential to define mass - e.g. Post. 1 in [4, Ftn. 1] — as was somehow noted by H. Hermes too - see [4, Ftn. 2 (b)]. Furthermore

\footnotetext{
${ }^{2}$ In connection with classical physics and special relativity, assertion $(E)$ is true (if "event" stands for "event-point"). However, Bressan regards event point as extensional in every (object) theory $\mathscr{T}$ of general relativity, and as a peculiar modal notion neither extensional nor absolute in the auxiliary semantical theory $\mathscr{S} \mathscr{T}$ associated with $\mathscr{T}$ - see from A4 to A6 in [4, pp. 56-58]
} 
$\eta)$ the existence postulate for mass-distributions on mass points, implicit in [4, Ftn. 1] but present in [1, p. 151] as Assioma 20, 1 (a)-(b), implies certain causal implications through Def. 1 in [4, Ftn. 1].

Let me add that, in ordinary English, "or" has two different interpretations and "possibility" has an even greater ambiguity; furthermore, asserting $(F)$ practically implies that in Bressan's axiomatic theories, " $\diamond p$ " may be interpreted as "it is logically possible that $p$ ". Should this interpretation actually be allowed, I would agree with the presence of "formal counterparts for" in $(A)$.

As for $(C)$ and $(D)$, I regard causal nexus as a very important explicandum in Carnap's sense. It is used in various senses within consideration about physical theories, e.g. in preliminaries or in view of applications. However (likely because of this ambiguity) it is used within no (object) theory of e.g. mathematical physics that I know. Furthermore,

७) my formal axiomatic systems were constructed, sometimes in various versions as in [4] and [5], to render the foundations of the Mach-Painlevé type for some physical theorie rigorous - see sect. 4 .

Therefore $(D)$ holds (so far).

3. In [3, pp. 200-201] one considers any sentence asserting that a certain phenomenon occurs in the space-time region $\mathcal{R}_{4}$; and for $r=1,2$ one (intuitively) explains the notions of causal and physical possibility : $^{\text {: see }} \gamma$ 's where " $r$ " stands for "in the $r$-th sense". E.g. " $p$ 's physical possibility 2 " briefly means $p$ 's compatibility with physical law. Furthermore the following is in effect stated.

A3.1 For $\mathcal{R}_{4}$ bounded, $p$ is said to be causally possible 1 if $p$ would be technically possible for suitable skilful technicians, ${ }^{3}$ i.e. if some technicians as skilful as some possible future technicians would be able to implement $p$ (by working outside $\mathcal{R}_{4}$ ), ${ }^{4}$ with an arbitrarily preassigned degree of approximation.

A3.2 For $\mathcal{R}_{4}$ unbounded, $p$ is said to be causally possible 1 if so is $p$ 's restriction to every bounded part of $\mathcal{R}_{4}$.

A3.3 Physical sentences that are causally possible 1 are said to be physically possible . $_{\text {. }}$.

\footnotetext{
${ }^{3}$ E.g. in [3] Bressan uses the natural notion of technical possibility, considered by Hutten in [8]. However, in [8] physical possibility is identified with physical possibility 2 , which in [3] is shown to have serious defects and to be fully unsatisfactory within foundations of the Mach-Painlevé type.

4 The above word "possible", absent from the corresponding sentence in [3], can also be referred to genetic engineering - see [4, Ftn. 3].
} 
Let us remark that in [3, pp. 200-201] a further explanation of physical possibilty 1 is given, mainly in classical physics; and besides avoiding some preceding counterfactual conditions, it affords a criterion to (somehow) check p's physical possibility experimentally. In fact this explanation uses an arbitrary physical proposition $p$ and a translation of it by the time $\tau(>0)$, say $p^{\prime}=p_{\tau}$; and it is based on the physical homogeneities of (classical) time and inertial spaces, as well as on the physical isotropy and mutual indistinguishability of these spaces if preferred.

Let us now consider the case where $p$ and $p^{\prime}$ are referred to two space-time frames $\mathcal{F}$ and $\mathcal{F}^{\prime}$ respectively (related to the same units of measure, possibly non-inertial, and) such that at all corresponding event-points $\mathcal{E} \in \mathcal{R}_{4}$ and $\mathcal{E}^{\prime}$ $\left(\in \mathcal{R}_{4}\right)$ the gravitational, dragging, and Cariolis' forces per unit mass have the same representations in $\mathcal{F}$ and $\mathcal{F}^{\prime}$ respectively. ${ }^{5}$ Then the afore-mentioned criterion in [3] has this extended version:

A3.4 (a) For $\mathcal{R}_{4}$ bounded, $p$ is (said to be) physical possible 1 if, for every approximation degree $\varepsilon(>0)$, some possible technicians - see Ftn. 4 - can implement some choice of $p^{\prime}=p_{\tau}$ (for $\tau$ large enough) within the approximation degree $\varepsilon$.

(b) the analogue of A3.2 for physical possibility 1 holds.

While the explanations A3.1-3 (in effect in [3]) for the notions of causal and physical possibilities also work quite well in the space-times of both special and general relativity, the version A3.4 of the afore-mentioned criterion in [3] has been considered because it can be extended to the latter space-time rather naturally unlike the original version.

In fact we can consider any physical assertions $p$ and $p^{\prime}$ that (i) express two conceivable phenomena occurring in the space-time regions $\mathcal{R}_{4}$ and $\mathcal{R}_{4}^{\prime}$, respectively, of general relativity and (ii) are such that the descriptions of $p$, $\mathcal{R}_{4}$, and $\mathcal{R}_{4}$ 's space-time metric in the former of some space-time frames $\mathcal{F}$ and $\mathcal{F}^{\prime}$ (related to the same units of measure) equal the analogous description for $p^{\prime}$ in $\mathcal{F}^{\prime}$. Now the following relativistic analogue of A3.4 can be stated.

A3.5 (a) For $\mathcal{R}_{4}$ bounded, $p$ is (said to be) physically possible 1 if, for every event point $\bar{\varepsilon}$ of the real space-time $\Sigma_{\rho}$, and for every approximation degree $\varepsilon(>0)$, some future (possible) technicians — see Ftn. 4 - are able to implement some choice of the above $p^{\prime}$ together with the space-time metric of $\mathcal{R}_{4}^{\prime}$, within the approximation degree $\varepsilon$ and with $\mathcal{R}_{4}^{\prime}$ belonging to $\bar{\varepsilon}$ 's posterior causal cone (in $\Sigma_{\rho}$ ).

(b) Part (b) of A3.4 holds.

\footnotetext{
${ }^{5}$ That $\mathcal{E}$ and $\mathcal{E}^{\prime}$ are corresponding event-points means here that they have the same coordinates in $\mathcal{F}$ and $\mathcal{F}^{\prime}$ respectively.
} 
The above criterion or explanation A3.5 is certainly more satisfactory than the one briefly written in $\left[4\right.$, Ftn. 4 , p. 55]. ${ }^{6}$ Incidentally the analogue of A3.5 for causal possibility and for $p$ belonging to a possibly indeterministic theory also appears to hold.

Instead the relativistic explanation written in [4, Ftn. 4] risks giving too weak a meaning to " $p$ 's physical possibilty 1 ". The same can be said for the explanation (of the same kind and belonging to classical physics) written in [4, Ftn. 3], as well as for the one introduced in [3, p. 200] with the words, "Perhaps some readers prefer $[\ldots]$ the following $[\ldots]$ ". ${ }^{7}$

4. As for the question raised in $(B)$ :

FIRST, I complete $(\vartheta)$ in sect. 2 by noting that in [1] I introduced my first formal framework and the first version of my axiomatization method ${ }^{8}$ to render Painlevé's foundations [10] for classical particle mechanics rigorous (and to extend it). This was done because:

८) a counterfactual conditional is (essentially) used in [10, p.65],

$\kappa)$ the axioms effectively written in [10] are not sufficient to prove all theorems asserted there - see [1, p. 106]; e.g., [10] includes nothing similar to the possibility axiom (Post. 1) mentioned in [4, Ftn. 1] in order to sketch a definition of mass, or to the existence axiom for mass-distributions (implying certain necessity assertions) hinted at in $\eta$ ), and

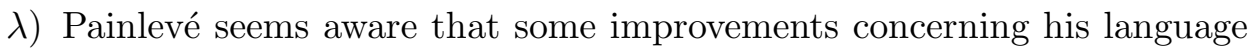
(hence formal logic) and axiomatic system might be useful or necessary. ${ }^{9}$

${ }^{6}$ The (brief) relativistic explanation in [4, Ftn. 4] reads in effect as:

A3.6 A proposition $p$ of any relativistic theory $\mathscr{T}$, that describes a (conceivable) phenomenon occurring in a space-time region $\mathcal{R}$ is said to be physically possible 1 if, for all $\mathcal{E} \in \mathcal{R}$, it is technically possible to implement a region $\mathcal{N}^{\prime}$ isometric with some (bounded) neighborhood $\mathcal{N}$ of $\mathcal{E}$, together with the transform $p_{\mathcal{N}, \mathcal{N}^{\prime}}$ by the above isometry, of $p$ 's restriction $p_{\mathcal{N}}$ to $\mathcal{N}$ (with an arbitrarily preassigned approximation).

7 Briefly speaking, as far as the explanation of " $p$ 's possibility 1 " is concerned, the afore-mentioned risk of A3.6 in Ftn. 6 seems avoided by requiring $p$ to describe a maximal conceivable evolution of the universe occurring in $\mathcal{R}$ (according to the physical theory being considered); and the same can be said of the afore-mentioned classical analogues of A3.6 in [4, Ftn. 3] and [3, p. 200].

8 The formal framework presented in [1] consists of an unusual extensional language capable of dealing with modal notions. This framework is used in $[4$, sect. 4$]$ in a generalized form.

9 As is more thoroughly stated in [2, Ftns. 52-53, p. 110], in [10, pp. 64-65] Painlevé writes "Je voudrais enoncer rapidement ce corps d'axiomes" and, after his counterfactual conditional referred to in $(\iota)$, "cette terminologie admise les axiomes [...] ce resument ainsi." 
$\mu)$ By $(\iota)$ and $(\lambda)$ I was pushed to use mathematical logic - see Ftn. 8 to solve the mathematical problems set in $(\kappa)$, just as mathematics is used to solve physical problems. Later I wrote the more complex modal theory in [2] to interpret the physical language of Painlevé more directly, and especially to analyze some notions involved by axiomatizations of the Mach-Painlevé type better.

Incidentally I note that the above considerations, especially $(\iota),(\lambda),(\mu)$ and Ftn. 9, serve to meet Urchs' request "for further explanation of how [my] proposal is related to what is really expressed in physical terminology by the notions [I exploit]" ([13, p. 41]) and that

$\nu$ ) paper [11], belonging to the axiomatization of biology, as well as [5] explicitly refer to my second formal framework [2], the one set in [1] being insufficient for their purposes (modalities serve in [11] to render some definitions and laws rigorous).

SECOND, Painlevé's foundations [10] have been used in several textbooks of rational mechanics. ${ }^{10}$ Furthermore - in compliance with $(\epsilon)$ and $(\eta)$ (sect. $2)$ - it is very easy to check $(\kappa)$, e.g. by reasoning similar to some essentiality considerations in [4, Ftn. 1] (see also [1, p. 106]). Lastly, essentially different rigorizations of [10] are not hinted at, as far as I know (see [4, Ftn. 2 (b)]).

By the First and SECOND points,

$\xi)$ Being [1] a rigorous version of [10], it constitutes, so to speak, an indirect positive answer to the question in $(B)$, and also an indirect "proof of reality" for Bressan's construction - see $\left(B^{\prime \prime}\right)$ in sect. 1 .

The following direct positive answers can be added. First, C. Truesdell, a well known mathematical physicist interested in axiomatizations admittedly not of the Mach-Painlevé type, shows a very favorable opinion regarding Bressan's axiomatic theory [1] — see [12, pp. 533-554] — while in [12, p. 532] he correctly refers to both [1] and [2] for other purposes.

Incidentally, in connection with $\left(B^{\prime}\right)$ in sect. 1 , it is interesting to note that Truesdell's considerations on [1] strongly agree with Garson's — see [7]; the latter is more interested in my interpreted formal framework, while the former explicitly notes that, e.g. I want to face "mathematical gaps", which is close to $(\mu)$.

Furthermore Truesdell contributed to and signed the presentation of Montanaro's paper labeled [193] in [4], which is in effect based on my formal system and involves some explicit modal axioms.

10 E.g. A. Signorini (Rome) and B. Finzi (Milan) followed [10], even if the latter regretted that thus some physical laws collapse into definitions. 
Another direct positive answer to the question in $(B)$ - and hence to the one on the "proof of reality" involved in $\left(B^{\prime \prime}\right)$ - is given by $(\nu)$, in that [11] was written and presented to the press at the initiative of some biologists.

I conclude by briefly noting that, e.g., the rigorization [1] of Painlevé's foundations [10] renders these more complex; and a larger complexity arises when one starts an extension of [1] to continuous media. ${ }^{11}$ However, even if this rigorization and extension decrease the possibility of divulgation, they have a scientific value, appearing from the preceding considerations. For example, even the aforementioned extension - implemented by, e.g. the papers [41], and [192] to [194] in [4] — can be useful to reach some concrete results, as it appears from the works [9] (a) and (b), as well as [6] - see Ftn. 1.

\section{References}

[1] Bressan, A., "Metodo di assiomatizzazione in senso stretto della Meccanica Classica. Applicazioni di esso ad alcuni problemi di assiomatizzazione non ancora completamente risolti", Rend. Sem. Mat. Univ. Padova 32 (1962), $55-212$.

[2] Bressan, A., A general interpreted modal calculus, Yale University Press (1972), 327 pp.

[3] Bressan, A., "On physical possibility", [in:] M. Luisa Dalla Chiara (ed.), Italian Studies in Philosophy of Science, Boston Studies in Phil. of Sci., Reidel (1981), 97-210.

[4] Bressan, A., "A semantical theory connected with relativistic space-time", [in:] Proceedings of the Symposium "Semantical Aspects of Space-Time Theories", Bielefeld, August 1991, edited by U. Majer and H. J. Schmidt, Wissenschaftsverlag, Leipzig (1994) 53-65.

[5] Bressan, A., "Again on relativistic semantics", Logic and Logical Philosophy, 3 (1995), 23-36.

[6] Bressan, A., and Montanaro A., "Towards the physical construction of two rigid heat-conducting bodies that are globally equivalent but physically different, with respect to small processes" (preprint).

[7] Garson, J. W., "Review of [1]", The Journal of Symbolic Logic, 38, 144.

[8] Hutten, E. H., The language of modern physics, London, G. Allen and Unwin, New York, Macmillan (1956).

11 Foundations of the Mach-Painlevé type for continuous media are not at all superfluous, because their reduction to particle systems is troublesome, unlike the converse reduction, which has in effect been well known for nearly a century (see [12, pp. 537-538]). 
[9] Montanaro, A., (a) "Global equivalence for deformable thermo-elastic bodies" (preprint), (b) "Global equivalence for rigid heat-conducting bodies" (preprint).

[10] Painlevé, P. Les axiomes de la mécanique, Paris, Gouthier-Villars (1992).

[11] Rizzotti, M. and Zanardo, A., "Assiomatizzazioni della genetica", Mem. Fis. Acc. Lincei, s. 9, v. 2 (1993), 87-125.

[12] Truesdell, C., An idiot's fugitive essays on science, Springer Verlag (1984), $645 \mathrm{pp}$.

[13] Urchs, M., "Comments on Aldo Bressan's paper", Logic and Logical Philosophy, 3 (1995), 37-42.

Aldo Bressan

Department of Mathematics

University of Padova

Via Belzoni 7

I-35131 Padova, ItALY 